\title{
Nonlinear structures in interchange mode turbulence
}

\author{
Sheikh Dastgeer, ${ }^{1, *}$ Raghvendra Singh, ${ }^{2}$ Hans Nordman, ${ }^{1}$ Jan Weiland, ${ }^{1}$ and André Rogister ${ }^{3}$ \\ ${ }^{1}$ Department of Electromagnetics, Chalmers University of Technology \& Euratom-VR Association, S-412 96 Göteborg, Sweden \\ ${ }^{2}$ Institute for Plasma Research, Bhat, Gandhinagar 382 428, India \\ ${ }^{3}$ Institut für Plasmaphysik der Forschungszentrum, Jülich, GmbH and Association EURATOM-KFA, Postfach 1913, \\ 5170 Jülich, Federal Republic of Germany
}

(Received 26 February 2002; revised manuscript received 6 June 2002; published 25 September 2002)

\begin{abstract}
Generation of linearly unstable streamers and nonlinearly saturated zonal flows have been investigated using two-dimensional simulations of interchange mode turbulence. It has been shown that nonlinear diamagnetic interactions tend to inhibit spectral cascades towards larger length scales and consequently suppress the nonlinear excitation of zonal flows. The latter has also been found to be in qualitative agreement with a theoretical analysis based on the reductive perturbation method.
\end{abstract}

DOI: 10.1103/PhysRevE.66.036408

PACS number(s): 52.35.Ra, 52.35.Mw, 52.35.Py

\section{INTRODUCTION}

Long wavelength structures such as streamers (radially extended, poloidally localized) and zonal flows (radially localized, poloidally extended) are widely considered to be important for governing transport and turbulence in magnetically confined toroidal plasmas. The implication of these structures for the understanding of anomalous plasma transport has thus received considerable attention in recent years. In the past, these structures have largely been pursued in the context of astrophysical, galactic plasmas [1-3] to understand the generation of large-scale zonal magnetic fields. The emergence of such large-scale highly anisotropic turbulent flows could be a manifestation of an inverse cascade phenomenon associated with the wave energy that gets transferred towards the long wavelength region [4-6]. In particular, within the context of tokamak plasmas, understanding of the generation of large-scale zonal or streamer flows has often been based on drift wave instabilities with Boltzmann electrons [7-9], and the flows have been attributed to Reynolds stresses [10], parametric and modulation instabilities of drift wave packets [11-14], and other mechanisms. In addition, other classes of drift wave instabilities, such as ion temperature gradient (ITG) modes, resistive drift ballooning modes, etc. have often been regarded as potential candidates for explaining the poloidal sheared flow in tokamaks and the formation of internal transport barriers (ITB) [15]. More realistic models consisting of a complete set of plasma fluids equations (density, temperature, vorticity, etc.), in the context of ITG modes, have also been analyzed in order to understand the formation of zonal flow structures [16] that have been previously reported in an earlier work [20]. Recently, a globally self-consistent ITG plasma model has been studied numerically to understand the poloidal zonal flows [18]. Sophisticated computer simulations, based on gyrokinetic and gyrofluid models, have also been helpful to deepen the understanding of such flows amidst a turbulent background of small-scale fluctuations and their mutual interplay [19-21].

In this paper, we examine the generation of low fre-

*Email address: dastgeer@elmagn.chalmers.se quency, large-scale streamers and zonal flows within the paradigm of flute-type interchange mode (IM) turbulence, wherein the ansatz of Boltzmann electrons has been replaced by the electron continuity equation. Additionally, ion temperature effects in the form of diamagnetic flows have been incorporated in the ion continuity equation and the linear diamagnetic frequency has been treated as a fixed parameter in the entire calculation. This is a reasonably simple model, which can be useful to describe the tokamak edge region, and, under suitable conditions, gives rise to the curvature driven mode, known as the Rayleigh-Taylor (RT) instability [22-24]. These instabilities (IM, RT, and their other manifestations) are not only invariably intrinsic to the tokamak plasmas confined by a strong magnetic field, but are also of importance in space plasmas. Moreover, the set of equations describing IM turbulence are essentially different from the drift wave models in terms of the nonlinear character. Unlike the latter, the nonlinear manifestations due to the simultaneous existence of convective $(\mathbf{E} \times \mathbf{B})$ and diamagnetic nonlinearities, and their interplay in the presence of an additional polarization nonlinearity, seem rather subtle and are more or less unexplored. These facts urge for a detailed exploration of the IM turbulence model and therefore form the prime motivation of our present investigations. Thus in the present work, we primarily concentrate on nonlinear properties of the IM turbulence and seek to understand the dynamics of zonal flows amidst such complex nonlinear interactions. The importance of diamagnetic nonlinearities in the generation of zonal flows has previously been reported in the context of ITG modes [9] where an increase of the Reynolds stress due to diamagnetic nonlinearities was found. Our investigations indicate that for IM turbulence, nonlinearly generated zonal flows may be radically suppressed by nonlinear diamagnetic interaction processes as a result of a reduction of turbulent wave energy cascades towards larger scales. The results can be explained in terms of the phase relations between density and potential perturbations obtained for IM and ITG turbulences; for ITG modes, $n$ and $\phi$ are in phase, resulting in an increase of the Reynolds stress forces; for interchange modes on the other hand, $n$ is out of phase with $\phi$ for small $\epsilon_{n}$ resulting in a reduction of the effective Reynolds drive.

The rest of the paper is organized as follows. In Sec. II, 


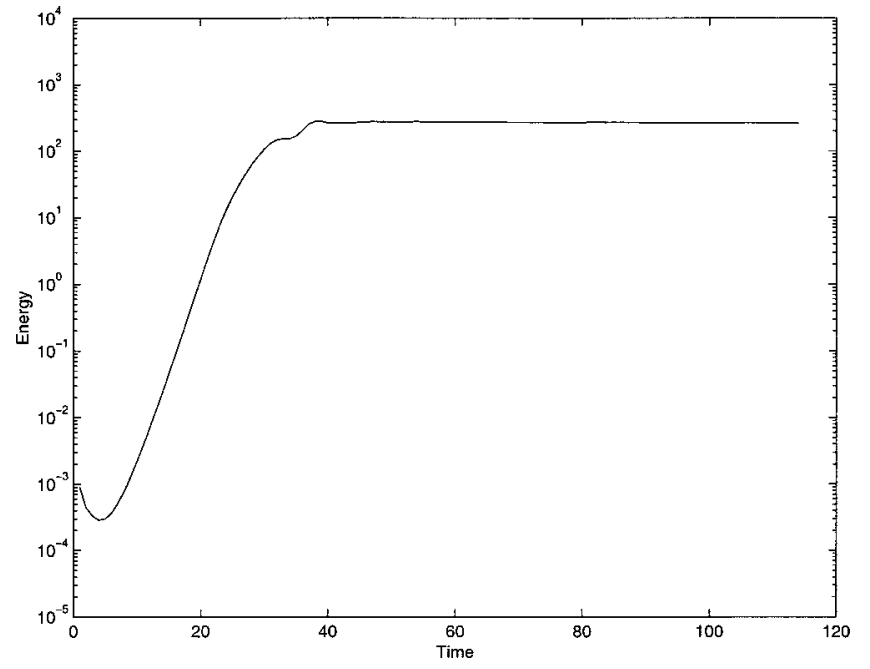

FIG. 1. Time evolution of total energy, i.e., $E=\int\left[n^{2}\right.$ $\left.+(\boldsymbol{\nabla} \phi)^{2}\right] d x d y$. Linear (up to $t=20$ ) and nonlinear phases of evolution are distinguishable.

governing equations of the IM turbulence model and their linear as well as nonlinear features have been presented. Section III contains results from numerical simulations, where we demonstrate how diamagnetic effects can alter nonlinear excitation of the zonal flows and suppress them. The theoretical understanding of the suppression of zonal flows has been carried out in Sec. IV, and Sec. V contains the discussion.

\section{BASIC EQUATIONS AND CONSERVATIONS}

We consider two-dimensional electrostatic interchange mode turbulence for a quasineutral, cold ion plasma immersed in a curved magnetic field. The basic equations can readily be cast in terms of two scalar variables, namely, den- sity and electrostatic potential;

$$
\begin{aligned}
& \frac{\partial n}{\partial t}+\epsilon_{n} \frac{\partial n}{\partial y}+\left(1-\epsilon_{n}\right) \frac{\partial \phi}{\partial y}+\hat{z} \times \nabla \phi \cdot \nabla n=D \nabla^{2} n \\
& \left(\frac{\partial}{\partial t}-\tau \frac{\partial}{\partial y}\right) \nabla^{2} \phi+\epsilon_{n}(1+\tau) \frac{\partial n}{\partial y}+\hat{z} \times \nabla \phi \cdot \nabla \nabla^{2}(\phi+\tau n) \\
& =\mu \nabla^{4} \phi .
\end{aligned}
$$

Equation (1) is the electron continuity equation, while Eq. (2) represents the ion vorticity equation. The diamagnetic (or FLR) terms are associated with the parameter $\tau=T_{i} / T_{e}$, the ratio of ion to electron temperatures, and appears in linear as well as in nonlinear terms. The other dimensionless variables and parameters, i.e., electrostatic potential, electron density, time, and spatial coordinates are, respectively, $\phi$ $=e \widetilde{\phi} L_{n} / T_{e} \rho_{s}, \quad n=\tilde{n} L_{n} / N \rho_{s}, \quad t=\tilde{t} L_{n} / C_{s}, \quad(x, y)$ $=(\bar{x}, \bar{y}) / \rho_{s}, \epsilon_{n}=2 L_{n} / R$; where $\epsilon, L_{n}, \rho_{s}, N, C_{s}, R, \mu$, and $D$ are, respectively, electric charge, density gradient length scale, ion Larmour radius, total density, sound speed, major radius of tokamak, viscosity, and particle diffusivity. The variables represented by tilde are the un-normalized variables. Equations (1) and (2) fulfill the following conservation law:

$$
\begin{gathered}
\frac{1}{2} \frac{\partial}{\partial t} \int\left[n^{2}+(|\nabla \phi|)^{2}\right] d x d y+\left(1+\epsilon_{n} \tau\right) \int n \frac{\partial \phi}{\partial y} d x d y \\
\quad=-\int\left[D(|\nabla n|)^{2}+\mu\left(\nabla^{2} \phi\right)^{2}\right] d x d y,
\end{gathered}
$$

which shows that the rate of change of total energy (pressure+ kinetic) decays due to flux, diffusivity, and viscosity effects. The linear eigenfrequency from Eqs. (1) and (2) reads as
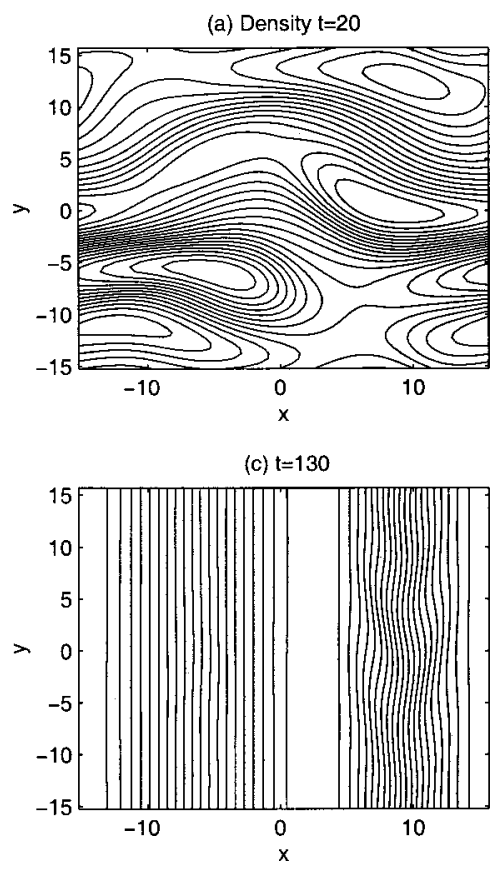

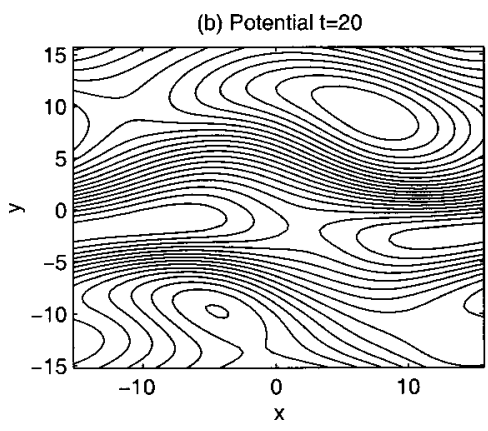

(d) $\mathrm{t}=\mathbf{1 3 0}$

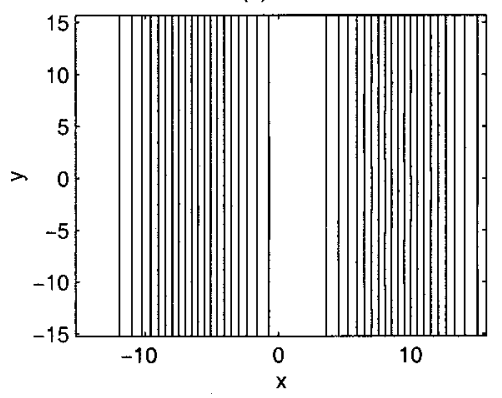

FIG. 2. Time evolution of linearly unstable streamers [(a) and (b) correspond to density and potential] and nonlinearly saturated zonal flows $\left[(\mathrm{c})\right.$ and (d)], for the parameters $\tau=0, \epsilon_{n}=0.8$, $\mu=D=0.1$. 


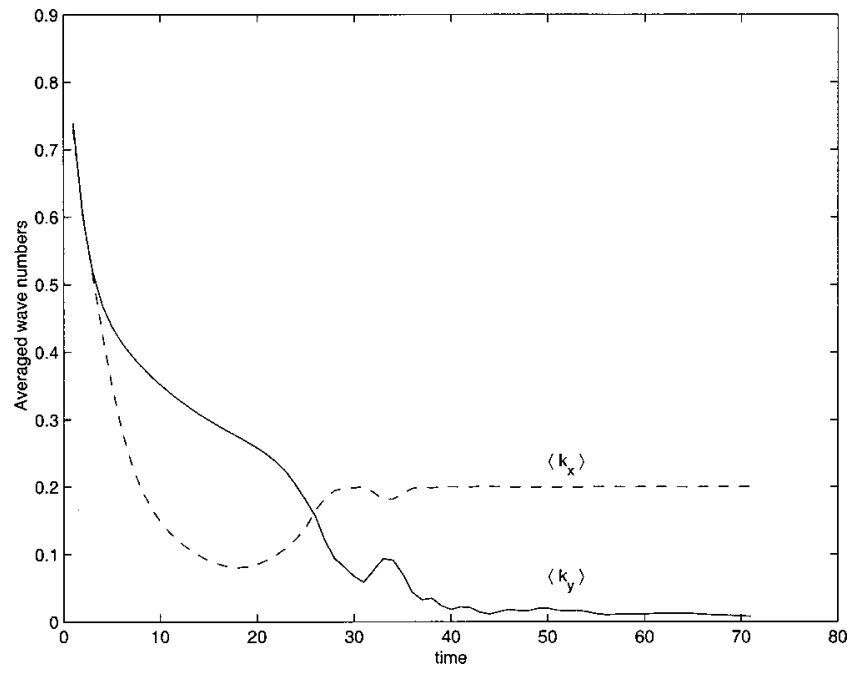

FIG. 3. Evolution of spectral averaged wave numbers defined as $\left\langle k_{Q}\right\rangle=\sqrt{\left(\Sigma_{k} k_{Q}^{2}\left|\phi_{k}\right|^{2}\right) /\left(\Sigma_{k}\left|\phi_{k}\right|^{2}\right)}$, where $Q=x$ or $y$. Initially the spectrum is highly isotropic as $\left\langle k_{x}\right\rangle=\left\langle k_{y}\right\rangle$. During the linear phase of evolution, sufficient anisotropy is developed in the turbulent spectrum due to streamers, thereby leading to $\left\langle k_{y}\right\rangle>\left\langle k_{x}\right\rangle$. While during the nonlinear phase, spectral transfer alters the turbulent spectrum leading to $\left\langle k_{y}\right\rangle\left\langle\left\langle k_{x}\right\rangle\right.$ due to generation of zonal flows.

$$
\begin{aligned}
\omega_{k}= & \frac{k_{y}}{2}\left(\epsilon_{n}-\tau\right)+i \frac{k_{y}}{k_{\perp}} \sqrt{\epsilon_{n}\left(1-\epsilon_{n}\right)(1+\tau)} \\
& \times\left\{\frac{k_{\perp}^{2}\left(\epsilon_{n}+\tau\right)^{2}}{4 \epsilon_{n}\left(1-\epsilon_{n}\right)(1+\tau)}-1\right\}^{1 / 2} .
\end{aligned}
$$

Here $k_{\perp}=\sqrt{k_{x}^{2}+k_{y}^{2}}$. It is worth noting that for positive real frequency (i.e., $\epsilon_{n}>\tau$ ), the modes propagate in the direction of the electron diamagnetic drift, while in the opposite case (i.e., $\epsilon_{n}<\tau$ ) they move along the ion diamagnetic drift direction. The dispersion relation further indicates that no linear

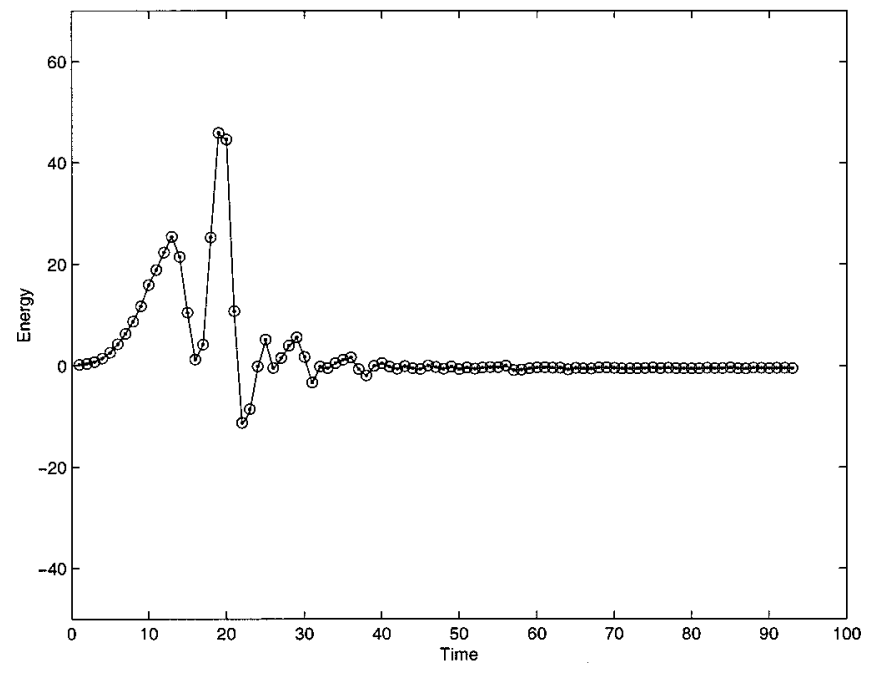

FIG. 4. Evolution of energy. The solid line represents the rate of change of total energy, i.e., $\frac{1}{2} \partial / \partial_{t} \int\left[n^{2}+(|\nabla \phi|)^{2}\right] d x d y$. Circles represent combination of energy flux and dissipative energy, i.e., $\int\left[\left(1+\epsilon_{n} \tau\right) n \partial \phi / \partial y+D(|\nabla n|)^{2}+\mu\left(\nabla^{2} \phi\right)^{2}\right] d x d y$. The figure clearly demonstrates a quantitative balance of the energy relation as given by Eq. (3), and so validates our numerical results. A constancy of the total energy is also very apparent here.

instability is likely to occur for the $k_{y}=0$ mode (essentially the zonal flow mode), while such a mode could possibly be generated via inverse cascading processes that are inherently governed by the nonlinear interactions. On the other hand finite $k_{y}$ modes could give rise to linear instabilities in the form of streamerlike structures $\left(k_{x}=0\right)$, which can be further reduced as a result of the linear diamagnetic term.

\section{RESULTS OF NUMERICAL SIMULATIONS}

Equations (1) and (2) have been integrated numerically using a fully dealiased pseudospectral method [24] in a two-

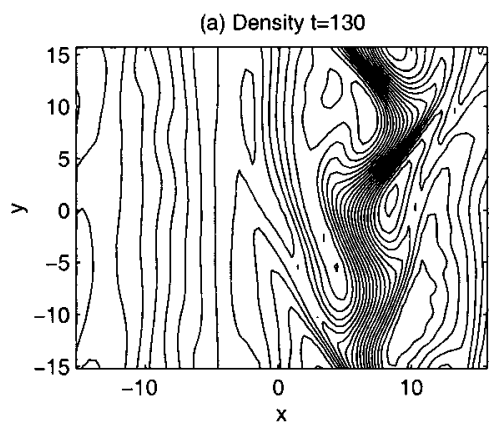

(c) Density $t=130$

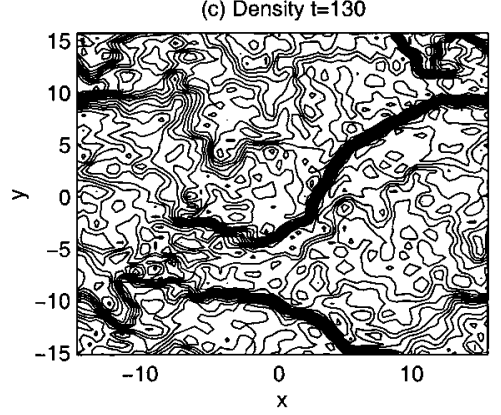

(b) Potential $\mathrm{t}=130$

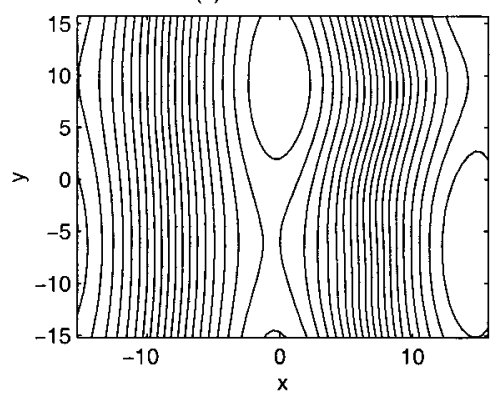

(d) Potential $t=130$

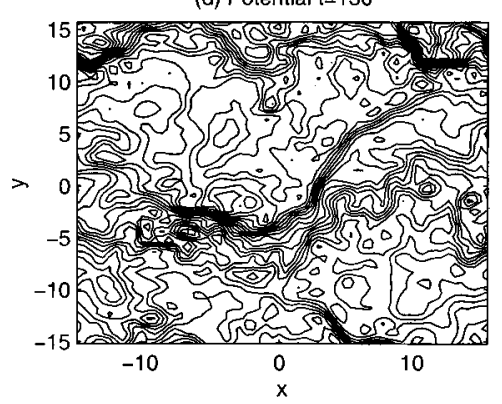

FIG. 5. (a) and (b) are saturated density and potential structures for $\tau=0.1$. The effects due to diamagnetic terms are distinguishable [compare with Figs. 1(c) and 1(d)]. The diamagnetic effects appear to damp out the zonal flow for $\tau$ $\left(=T_{i} / T_{e}\right)=1.0$, as can be seen in (c) and (d). 


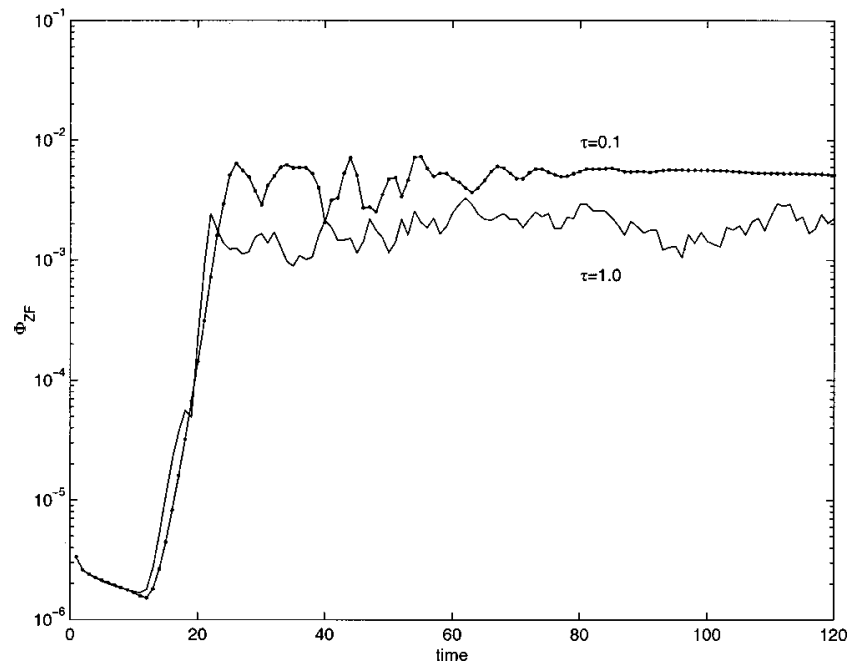

FIG. 6. Time evolution of the $\Phi_{\mathrm{ZF}}$ (zonal flow) mode. The initial excitation amplitude is observed to be strongly reduced for an increasing value of $\tau, \epsilon_{n}=0.2$.

dimensional box of $N^{2}$ Fourier modes, with doubly periodic boundary conditions along the $x$ and $y$ directions. This method uses discrete Fourier representation in which the linear part of the equations is integrated exactly. The nonlinear terms, on the other hand, are evaluated in real space. The variables are then transformed from real to Fourier space and back, using fast Fourier transform (FFT) routines at each time step. The time evolution is taken care of by a leap-frog predictor corrector scheme. The accuracy of the numerical simulation results has been ensured by continuously monitoring the energy integrals [Eq. (3)] at each time step. The initial spectrum of perturbed density and potential is comprised of discrete Fourier modes that are highly uncorrelated and randomly phased, thereby ensuring a perfectly isotropic turbulent spectrum. We here basically concentrate on the nonlinear evolution of such a spectrum under the influence of increasingly complex diamagnetic interactions as $\tau$ increases and investigate how they influence the nonlinear structures. In order to gain an appropriate understanding of the role of nonlinear diamagnetic interactions, simulations with and without diamagnetic terms included were performed and compared. The entire evolution consists of two distinct phases, the linearly unstable phase wherein the streamers are generated followed by the nonlinear saturation, which eventually leads to a steady-state nonlinear structure namely, zonal flows. In Fig. 1 the time evolution of Eqs. (1) and (2) with $\tau=0$ is illustrated, which distinctively shows the linear (until $t=20)$ as well as the nonlinear $(t>20)$ behavior. Correspondingly, Figs. 2(a) and 2(b) indeed display persistence of the linear streamers $\left(k_{x} \simeq 0\right)$ emerging out of isodensity and potential contours until $t=20$ (end of the linear phase of evolution). The nonlinear interactions are likely to become significant thereafter, and tend to hinder energy cascades towards smaller $k_{x}$. Simultaneously there appears to be substantial transfer of energy towards the $k_{y}=0$ mode, leading to the formation of zonal flow structures, as shown in Figs. 2(c) and 2(d) (respectively, fluid density and potential structures). It is worth noting here that the density and potential

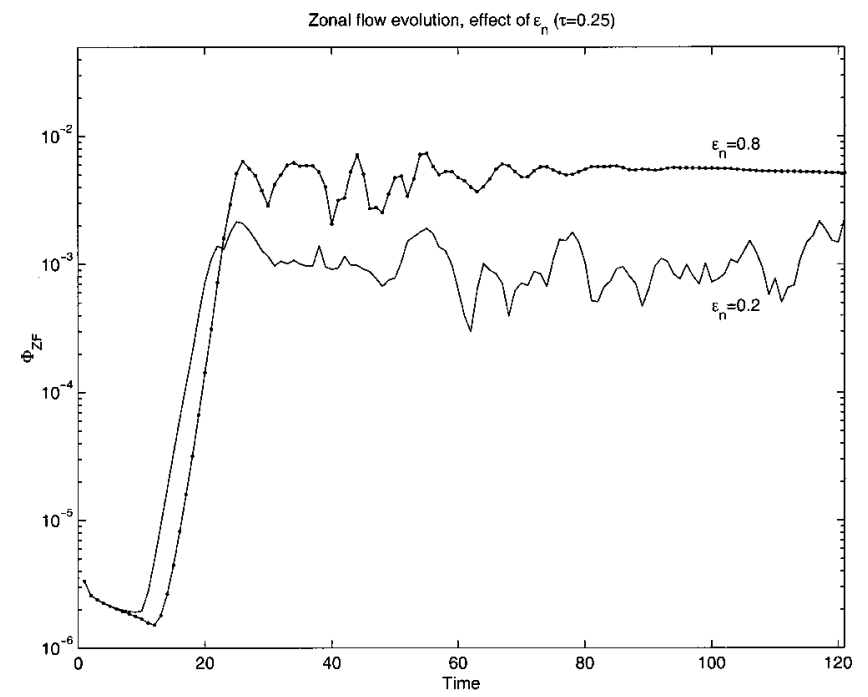

FIG. 7. Time evolution of $\Phi_{\mathrm{ZF}}$, the zonal flow component for $\epsilon_{n}>\tau$ and $\epsilon_{n}<\tau$, represented, respectively, by the upper and lower curves.

fluctuations tend to develop sufficiently anisotropic spectra during linear as well as nonlinear saturated states in spite of the fact that the choice of initial [highly isotropic to begin with, i.e., $\left\langle k_{x}\right\rangle \simeq\left\langle k_{y}\right\rangle$ at $t=0$ and the wave numbers $k_{x}, k_{y}$ remain symmetric during the early phase of evolution, see Fig. 3] as well as boundary conditions (periodic in $x, y$ directions) did not impose any kind of anisotropy. Moreover, these results are independent of the size of computational domain, number of Fourier modes, as well as the integration time step. We have carefully confirmed the simulation results for different random initial conditions, computational box sizes $(10 \pi \times 10 \pi, 15 \pi \times 15 \pi$, and $20 \pi \times 20 \pi)$, Fourier modes $\left(64^{2}, 128^{2}\right.$, and $\left.256^{2}\right)$, and the integration time steps $\left(1 \times 10^{-4}, 5 \times 10^{-4}\right.$, and $\left.4 \times 10^{-3}\right)$. A quantitative balance of the rate of change of total energy from Eq. (3) verifies our simulation results. A typical case shown in the Fig. 4 is basically run for $128^{2}$ Fourier modes in the two-dimensional box of size $10 \pi$ in each direction, for the time step $4 \times 10^{-3}$. The anisotropic zonal flows in our simulation have been defined as an average of the potential fluctuations over all the modes when $k_{y} \simeq 0$.

We next investigate the effect of an increasing influence of diamagnetic interactions on the dynamical evolution of zonal flows. Interestingly, even for moderately low values of $\tau(<1)$, the nonlinear zonal flows are found to be strongly reduced. This can be seen in Figs. 5(a) and 5(b). Moreover, for appreciably large value of $\tau(\geqslant 1)$, the zonal flows are no longer stable and smear off into several small scales structures [see Figs. 5(c) and 5(d)]. A more quantitative illustration of the suppression of zonal flows due to diamagnetic effects can be seen in Fig. 6, which clearly exhibits how the initial excitation level of zonal flows (which begins after $t$ $=20$ ) strongly declines. It can also be seen here that for $\epsilon_{n}$ $>\tau$, the zonal flows persist (top curve in Fig. 6) due to the increased Reynolds drive. On the other hand, when $\epsilon_{n}<\tau$ the zonal flows are relatively suppressed (see the bottom curve in Fig. 6). The results can be explained in terms of the 
density-potential correlation, as follows. In the latter case the phase relation between $n$ and $\phi$ is modified, i.e., $n$ $\simeq\left(\omega_{*} / \omega_{r}\right) \phi$, where $\omega_{r}<0$ for $\epsilon_{n}<\tau$ resulting in a reduction in the effective Reynolds stress forces [see nonlinear terms of Eq. (2); $\left.\hat{z} \times \boldsymbol{\nabla} \phi \cdot \nabla \nabla^{2}(\phi+\tau n)\right]$. The existence of zonal flows subject to the condition $\epsilon_{n}>\tau$ can also be verified in our simulation by fixing the parameter $\tau$ and varying $\epsilon_{n}$. This has been confirmed for two extreme cases of $\epsilon_{n}$, and is shown in the Fig. 7. The top and bottom curves in the figure represent the cases $\epsilon_{n}>\tau$ and $\epsilon_{n}<\tau$. It can be clearly seen that zonal flows have been enhanced for $\epsilon_{n}>\tau$, while they are suppressed in the opposite limit, which is consistent with our earlier observations. Furthermore, the spiky oscillation in the temporal evolution of the zonal flow for finite diamagnetic interactions is profound. Similar behavior was also noticeable in the recent gyrokinetic simulation of ITG mode turbulence [25]. Moreover, the long time evolution in our simulations indicates that the turbulence as well as the zonal flows reach a nonlinearity saturated state (see Figs. 1, 6 , and 7).

The suppression of zonal flow due to nonlinear diamagnetic interactions is identified in our simulations as an inhibition of the turbulent energy cascading towards the smaller wave numbers in the poloidal direction, which is directly proportional to the intensity of the nonlinear diamagnetic interactions. Our investigations further confirm that the diamagnetic nonlinearity (i.e., $\tau \hat{z} \times \boldsymbol{\nabla} \phi \cdot \nabla \nabla^{2} n$ ) is primarily responsible for counteracting the conventional polarization nonlinearity in the ion vorticity equation. The latter is known to cascade turbulent energy basically towards smaller wave numbers.

\section{THEORETICAL ANALYSIS}

In order to understand the mechanism leading to the suppression of nonlinearly saturated zonal flows, as observed in simulations, we provide a theoretical analysis by investigating the effect of the diamagnetic terms on the nonlinear zonal flow modes of zero frequency and with $k_{y}=0$. A reductive perturbation method [27] has been applied to the set of Eqs. (1) and (2). Using this method, we deduce the saturated potential of zero frequency, which is essentially a zonal flow. The variables can be expanded in spherical harmonics using $\Theta=\Sigma_{\alpha} \varepsilon^{\alpha} \Theta^{(\alpha)}$, with $\Theta^{(\alpha)}=\Sigma_{\ell} \Theta_{\ell}^{(\alpha)}(x, \xi, T) \exp \left[i \ell\left(k_{y} y\right.\right.$ $-\omega t)$ ], where $\Theta$ corresponds to the dynamical density $(n)$ and potential $(\phi)$ variables, $\varepsilon$ is the parameter characterizing smallness of the amplitude of the variables, $\xi=\varepsilon(y-u t)$, and $T=\varepsilon^{2} t$. The particle diffusivity $D$ and viscosity $\mu$ order as $\sim\left(\varepsilon^{2}\right)$. The amplitudes are subject to the reality condition as $\Theta_{\ell}^{(\alpha)}=\Theta_{-\ell}^{(\alpha)^{*}}$ and $\Theta_{\ell}^{(1)}=0$ for $\ell= \pm 1$. We thus consider the nonlinear modulation of a quasimonochromatic interchange mode. The first-order $\varepsilon^{1}$ equation then readily yields

$$
i D_{\ell}(\omega, k) \phi_{\ell}^{(1)}=0 \text {, }
$$

which is the dispersion relation for $\ell=1$. In arriving at the above relation, we have assumed the sinusoidal dependence of the perturbed variables as $\phi_{\ell}^{(1)}=\widetilde{\phi}_{\ell}^{(1)}(\xi, T) \sin k_{m} x$, and $n_{\ell}^{(1)}=\widetilde{n}_{\ell}^{(1)}(\xi, T) \sin k_{m} x$, where $k_{m}=m(2 \pi / L) \quad(m$
$=1,2, \ldots)$ and $L$ is the length of the system. For $\ell=1$, it leads to $D_{1}=0$, as $\phi_{l}^{(1)} \neq 0$ which is the dispersion relation for the interchange mode instability, as described earlier. The second-order $\varepsilon^{2}$ equations from Eqs. (1) and (2) may be written as

$$
i D_{\ell}(\omega, k) \phi_{\ell}^{(2)}+\zeta_{\ell} \frac{\partial \phi_{\ell}^{(1)}}{\partial \xi}=0
$$

where

$$
\begin{aligned}
\zeta_{\ell}= & \omega \ell^{2} k_{y}+2 \ell^{2} k_{y}^{2}-\Delta u-\tau+\epsilon_{n}(1+\tau) \alpha \\
& +\left[1-\frac{\left(\epsilon_{n}-u\right) k_{y}}{\left(-\omega+\epsilon_{n} k_{y}\right)}\right]\left(1-\epsilon_{n}\right),
\end{aligned}
$$

$\Delta=\partial^{2} / \partial x^{2}-\ell^{2} k_{y}^{2}=-\left(k_{m}^{2}+\ell^{2} k_{y}^{2}\right), \quad \alpha=\left(1-\epsilon_{n}\right) k_{y} /(\omega$ $\left.-\epsilon_{n} k_{y}\right)$. Since $D_{1} \rightarrow 0$ for $\ell=1$, hence $\zeta_{1}=0$ as $\partial \phi_{1}^{(1)} / \partial \xi$ $\neq 0$, which consequently leads to the expression for the group velocity $\left(u=\partial \omega / \partial k_{y}\right)$ of the interchange modes.

In the third-order $\left(\varepsilon^{3}\right)$, we obtain nonlinear equation as follows:

$$
\begin{aligned}
\frac{\partial n_{\ell}^{(1)}}{\partial T} & +\left(\epsilon_{n}-u\right) \frac{\partial n_{\ell}^{(2)}}{\partial \xi}+\left(1-\epsilon_{n}\right) \frac{\partial \phi_{\ell}^{(2)}}{\partial \xi} \\
& +i \ell\left[\left(-\omega+\epsilon_{n} k_{y}\right) n_{\ell}^{(3)}+\left(1-\epsilon_{n}\right) k_{y} \phi_{\ell}^{(3)}\right] \\
= & i \ell k_{y}\left[\phi_{\ell}^{(1)} \frac{\partial n_{0}^{(2)}}{\partial x}-n_{\ell}^{(1)} \frac{\partial \phi_{0}^{(2)}}{\partial x}\right] \\
& +\left(\alpha^{*}-\alpha\right) \frac{\partial}{\partial x} \frac{\partial}{\partial \xi}\left|\phi_{1}^{(1)}\right|^{2}
\end{aligned}
$$

$$
\begin{aligned}
\Lambda \frac{\partial \phi_{l}^{(1)}}{\partial T}+i \ell\left\{-\Lambda\left(\omega+\tau k_{y}\right) \phi_{\ell}^{(3)}+\epsilon_{n}(1+\tau) k_{y} n_{\ell}^{(3)}\right\} \\
\quad-i \ell\left[\left(\omega+2 u k_{y}\right)+3 \tau k_{y}\right] \frac{\partial^{2} \phi_{\ell}^{(1)}}{\partial \xi^{2}} \\
\quad-\left\{\Lambda(u+\tau)+2 \ell\left(\ell k_{y}-\omega\right)\right\} \frac{\partial \phi_{\ell}^{(2)}}{\partial \xi}+\epsilon_{n}(1+\tau) \frac{\partial n_{\ell}^{(2)}}{\partial \xi} \\
=i \ell k_{y}\left\{\phi_{\ell}^{(1)} \frac{\partial^{3}}{\partial x^{3}}\left(\phi_{0}^{(2)}+\tau n_{0}^{(2)}\right)-\Lambda\left(\phi_{\ell}^{(1)}+\tau n_{\ell}^{(1)}\right) \frac{\partial \phi_{0}^{(2)}}{\partial x}\right\} \\
\quad+2 k_{y}^{2}\left[1+\tau\left(\alpha^{*}+\alpha\right)\right] \frac{\partial}{\partial x} \frac{\partial}{\partial \xi}\left|\phi_{1}^{(1)}\right|^{2},
\end{aligned}
$$

where $\Lambda=\left(\partial^{2} / \partial x^{2}-k_{y}^{2} \ell^{2}\right)$ and $\Theta_{\ell}^{(2)} \sim \sin k_{m} x$. The terms associated with the particle diffusion $D$ and viscosity $\mu$ do not appear in the above equations as they are higher-order terms, and as such are neglected. While arriving at Eqs. (4) and (5), the nonlinear interaction mechanism tends to generate a few more terms corresponding to the fluxes in electron density and ion vorticity equations. These nonlinear fluxes are expected to be balanced by the corresponding sources or sinks 


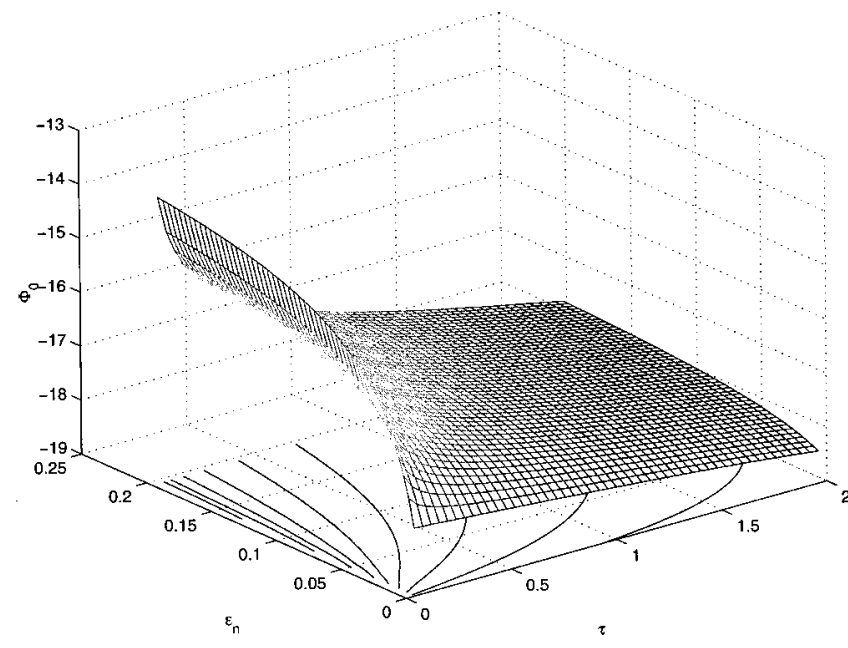

FIG. 8. Zero-frequency component zonal flow $\Phi_{0}$, as a function of $\tau, \epsilon_{n}$, as obtained from theoretical analysis.

in the respective equations. From Eqs. (4) and (5), the zero frequency component flow can readily be determined by putting $\ell=0$,

$$
\begin{aligned}
\phi_{0}^{(2)}= & \frac{2 k_{y}^{2}-\epsilon_{n}\left(\frac{\alpha-\alpha^{*}}{u-\epsilon_{n}}\right)-\tau\left\{\frac{\alpha-\alpha^{*}}{u-\epsilon_{n}} \epsilon_{n}-2 k_{y}^{2}\left(\alpha+\alpha^{*}\right)\right\}}{4 k_{m}^{2}+\epsilon_{n}\left(\frac{\alpha-\alpha^{*}}{u-\epsilon_{n}}\right)+\tau\left\{4 k_{m}^{2}+\epsilon_{n}\left(\frac{\alpha-\alpha^{*}}{u-\epsilon_{n}}\right)\right\}} \\
& \times \frac{\partial}{\partial x}\left|\phi_{1}^{(1)}\right|^{2} .
\end{aligned}
$$

The nonlinearly saturated component of the flow $\left(\phi_{0}^{(2)}\right)$, i.e., the zonal flow, is therefore generated by the pondermotive forces that are proportional to $\sim\left|\phi_{1}^{(1)}\right|^{2}$. The nonlinear mode coupling is assumed to saturate the interchange mode instability and hence the turbulent fluctuations, in the saturated state, can be estimated as [26] $\phi_{1} \simeq\left(\gamma / \omega_{d}\right) /\left(k_{m} L_{n}\right)$, where $\omega_{d}$ is the magnetic drift frequency. Writing growth rate of the interchange mode turbulence as $\gamma=\sqrt{\omega \omega_{d}}$, and $L_{n} \sim \epsilon_{n}$ for a fixed $R$, where $R$ is the major radius of the tokamak, the turbulent saturation amplitude can be written as $\phi_{1}$ $\sim \sqrt{\omega / \omega_{d}} /\left(k_{m} \epsilon_{n}\right)$. Using this estimation in Eq. (6) within the reasonable parameter range of $\left(\epsilon_{n}, \tau\right)$, the variation of $\phi_{0}^{(2)}$, essentially the zonal flow component, is shown in Fig. 8. This indeed shows the suppression of zonal flows when the diamagnetic effects become stronger (i.e., $\tau>0$ ); thus, demonstrating qualitative agreement with our simulation results as described earlier. Moreover, the amplitude of the saturated zonal flow enhances with increasing $\epsilon_{n}$ (for finite $\tau$ value) as seen in the analytical treatment, and this qualitatively agrees with the simulation results.

We further point out that our theoretical analysis (i.e., Fig. 8) supports the arguments that have been made earlier in Sec.
I with regard to the suppression or enhancement of the zonal flows in the IM turbulence model. It is clear from Fig. 8 that there could be strong excitation of the zonal flows when $\epsilon_{n}$ $>\tau$, for which $n$ and $\phi$ are in phase since $\omega_{r}>0$. Contrary to this, in the regime when $\epsilon_{n}<\tau$, there is remarkable suppression of the saturated zonal flow component in Fig. 8 due to the fact that density and potential fluctuations are now out of phase on account of the negative real frequency of the IM modes, which possibly reduces the Reynolds drive.

\section{CONCLUSION}

It has been identified in the present work that diamagnetic interactions can indeed alter the nonlinear cascading properties of the turbulent energy towards larger length scales in interchange mode turbulence. This, in turn, leads to a strong degradation of the zero-frequency component zonal flow. While the polarization nonlinear term conventionally cascades energy towards larger scales, the diamagnetic nonlinearity is observed to reduce the energy cascades towards larger length scales. It is to be noted that the linear diamagnetic term in our local simulation does not change with the radial distance.

In conclusion, the main emphasis here is to explore the effect of nonlinear diamagnetic effects on the generation of zonal flows. We discover that nonlinear diamagnetic effects counteract the usual polarization nonlinear effects (responsible for the Reynolds drive) resulting in the damping of the zonal flows. The underlying mechanism of zonal flow suppression observed here critically depends upon the phases of the density and potential fluctuations of the interchange modes. The results indicate the importance of finite ion temperature effects for interchange mode turbulence, even in situations where $T_{e} \gg T_{i}$. To draw more detailed conclusions, a more realistic description of the ion temperature fluctuations, including effects of finite $\eta_{i}$ (essentially a ratio of density and temperature gradient length scales), is needed in the simulation model. The present simulation is based on a local analysis where the profile parameters, including the diamagnetic drift terms, are held fixed in the simulation box. A global simulation including a radial dependence of the diamagnetic drift could potentially alter our local results. For instance, earlier work shows that an inhomogeneous diamagnetic frequency can introduce a radial inhomogeneity associated with the radially propagating waves in the system, which may reduce Reynolds stress forces and hence zonal flows [17]. This is left for future investigations.

\section{ACKNOWLEDGMENT}

One of the authors (R.S.) gratefully acknowledges the kind support and hospitality of Chalmers University of Technology, Göteborg, Sweden. 
[1] T. Soomere, Phys. Rev. Lett. 75, 2440 (1995).

[2] E. R. Priest, Solar Magnetohydrodynamics (Reidel, London, 1982), p. 15.

[3] R. Thompson, J. Atmos. Sci. 27, 1107 (1970).

[4] R. Z. Sagdeev, V. D. Shapiro, and V. I. Shevchenko, Sov. J. Plasma Phys. 4, 551 (1978).

[5] A. Rogister and G. Hasselberg, Phys. Rev. Lett. 48, 249 (1982).

[6] A. Hasegawa and M. Wakatani, Phys. Rev. Lett. 59, 1581 (1987).

[7] A. I. Smolyakov, P. H. Diamond, and M. Malkov, Phys. Rev. Lett. 84, 491 (2000).

[8] A. I. Smolyakov, P. H. Diamond, and V. I. Shevchenko, Phys. Plasmas 5, 1349 (2000).

[9] A. I. Smolyakov, P. H. Diamond, and M. V. Medvedev, Phys. Plasmas 7, 3987 (2000).

[10] P. H. Diamond and Y. B. Kim, Phys. Fluids B 3, 1626 (1991).

[11] P. N. Guzdar, Phys. Plasmas 2, 4174 (1995).

[12] P. N. Guzdar and R. G. Kleva, Liu Chen, Phys. Plasmas 8, 459 (2001).

[13] Liu Chen, Zhihong Lin, and Roscoe White, Phys. Plasmas 7, 3129 (2000).

[14] J. F. Drake, J. M. Finn, P. Guzdar, V. Shapiro, V. Shevchenko, F. Waelboeck, A. B. Hassam, C. S. Liu, and R. Sagdeev, Phys.
Fluids B 4, 488 (1992).

[15] K. H. Burrel, Phys. Plasmas 4, 1499 (1997).

[16] Sangeeta Mahajan and Jan Weiland, Plasma Phys. Controlled Fusion 42, 987 (2000).

[17] A. M. Dimits, et al., Phys. Plasmas 7, 969 (2000).

[18] S. Dastgeer, J. Weiland, and S. Mahajan, Phys. Plasmas 9, 1565 (2002).

[19] A. Dimits, T. J. Williams, J. A. Byers, and B. I. Cohen, Phys. Rev. Lett. 77, 71 (1996).

[20] Z. Lin, T. S. Hahm, W. W. Lee, W. M. Tang, and R. B. White, Science 281, 1835 (1998).

[21] G. Hammett, M. Beer, W. Dorland, S. C. Cowley, and S. A. Smith, Plasma Phys. Controlled Fusion 35, 937 (1993).

[22] V. P. Pavlenko and J. Weiland, Phys. Fluids 23, 719 (1980).

[23] J. M. Finn, J. F. Drake, and P. N. Guzdar, Phys. Fluids B 4, 2758 (1992).

[24] Amita Das, Sangeeta Mahajan, Predhiman Kaw, and Abhijeet Sen, S. Benkadda, A. Verga, Phys. Plasmas 4, 1018 (1997).

[25] Z. Lin, T. S. Hahm, W. W. Lee, W. M. Tang, and R. B. White, Phys. Plasmas 7, 1857 (2000).

[26] H. Nordman, J. Weiland, and A. Jarmen, Nucl. Fusion 30, 983 (1990).

[27] K. Nozaki, T. Taniuti, and K. Watanabe, J. Phys. Soc. Jpn. 46, 983 (1979); 46, 991 (1979). 